\title{
Occurrence and Distribution of Entomopathogenic Fungi in Agricultural Soil of Durg District of Chhattisgarh, India
}

\author{
Jumade Pratibha*, S. Pal and P.K. Sanyal \\ Department of Veterinary Parasitology, College of Veterinary Science \& Animal Husbandry, \\ Chhattisgarh Kamdhenu Vishwavidyalaya, Anjora, Durg, India \\ *Corresponding author
}

\section{Keywords}

Biological control, Entomopathogenic, fungus, Soil samples, Ticks

\section{Article Info}

\section{Accepted:}

11 June 2020

Available Online:

10 July 2020

\section{A B S T R A C T}

Entomopathogenic fungi are important natural enemies of arthropods and can be used for biological control of ticks. They are widely distributed in a wide range of habitats including aquatic, forest, agricultural soil and pasture habitats. Sixty crop roots soil samples were collected from nearby field and grazing areas of animals in Durg district of Chhattisgarh. A total of seven fungal isolates were recovered from the organic environment of Durg, Chhattisgarh and they were belonging to Genus; Aspergillus, Penicilium, Cladosporium, Fusarium, Rhizopus, Metarhizium and Trichoderma. The fungal colonies were isolated from soil samples and surface sterilized ticks were treated with aqueous fungal suspension. The mortality of ticks was observed on third to seventh day when hyphae covered the whole body surface. Out of the seven isolates explored from soil samples, four isolates were found to infect ticks namely, Fusarium sp., Rhizopus sp., Metarhizium sp. and Trichoderm sp.

\section{Introduction}

Tick and tick borne diseases are one of the biggest public health and veterinary problems in the world. These ectoparasites have an impact on the production and health of the animals through sucking blood or by transmitting the infectious agents such as viruses, bacteria, rickettsiae and protozoa (Eskezia and Desta, 2016).
Use of Chemical acaricides for control of ticks was considered as one of the best methods, but ticks have developed resistance against a range of currently-used acaricides such as organophosphates, carbamates, synthetic pyrethroids and amidines (Martins et al., 1995). The use of insecticides also produces environmental pollution with residues in milk, meat, vegetables and producing detrimental effects to human health. Biological control of ticks using 
entomopathogenic fungus is proved to be most economical and safest method to overcome the risk of environmental pollution and acaricidal resistance (Lacey et al., 2001). The bio-control potentials of entomopathogenic fungi (EPF) against animal ticks have shown promising results (Rao and Narladkar, 2017). The ability of EPF to penetrate the cuticle of arthropods, to kill several stages of the same pest and the relatively specific virulence of a single strain to one or a small group of pests make them good candidates as biocontrol agents (Samish et al., 2004). The present work was aimed to isolate and characterize the EPF from agricultural soil of Durg District in Chhattisgarh.

\section{Materials and Methods}

\section{Isolation and morphological studies}

Soil samples were collected during the period from September, 2018 to October, 2019 from ten villages namely Anjora, Rasmada, Thanaud, Khapri, Sriloda, Nagpura, Damoda, Dhaba, Dhanora and Mohlai. From each village, six fields which were under cultivation of either paddy or maize or seasonal vegetable crops were selected for collection of soil samples. The distance between two villages was about 3 to 5 kilometers and the distance between two fields was about half to 1 kilometer. A total of Sixty soil samples of 25 gram each were collected in zipped polythene bags from sampling point nearby crop plant root i.e. from the area with $5 \mathrm{~cm}$ in diameter and $5 \mathrm{~cm}$ deep around the crops using a trowel with a total area of $16 \mathrm{~m}^{2}$ from each field as per the method described by Amy et al., (2009). The soil samples were sieved through $2 \mathrm{~mm}$ mesh size to remove course material. The isolation of fungi from soil samples was enumerated by using serial soil dilution and soil plate method (Waksman, 1922) on Potato Dextrose Agar
medium.Four serial dilution of soil samples were undertaken in $15 \mathrm{ml}$ test tubes. Dilutions of $10^{2}, 10^{3}$ and $10^{4}$ were made to avoid overcrowding of fungal colonies. Potato Dextrose Agar Medium was prepared at a final concentration of $2.5 \%$ in conical flasks and autoclaved. Antibiotic solution using tetracycline and neomycin (w/v) was added to the medium at the rate of $0.02 \%$ after autoclaving to suppress bacterial growth. The molten medium was poured in radiation sterilized Petri plates $(90 \mathrm{~mm})$ in aseptic condition and allowed to solidify. One $\mathrm{ml}$ of the suspension of soil sample of each concentration was added to sterile Petri plates, in triplicates of each dilution, containing sterile Potato Dextrose Agar medium using micropipette. The plates were gently rotated to disperse the sample uniformly on agar plate. The plates were then incubated at $29^{\circ} \mathrm{C}$ and $75 \%$ relative humidity for 7 days. Plates were regularly monitored for fungal surface colonies. One isolate of each fungal growth from each soil sample was selected at random and further sub cultured. The subcultures were maintained on Potato Dextrose Agar Slants.

The ticks collected from the body of animals were rinsed with distilled water and then treated with $1 \%$ Potassium hypochlorite solution to prevent bacterial contamination. The surface sterile ticks were then placed on each fungal isolate and observed for fungal growth. The fungal isolates infecting ticks were separated and assumed as EPF. Isolation of entomopathogenic fungi was also done by placing the sterile ticks on petri plates containing soil sample. The soil samples were uniformly spread on Petri plates and moisture was maintained by adding sterile distilled water. Then sterile ticks were placed on soil samples and regularly observed for growth of fungal colonies. Ticks infected with fungus were isolated and then placed on petri plates containing growth media. The fungal colonies 
were then further subcultured to obtain the pure culture.

\section{Results and Discussion}

Out of the sixty soil samples collected from agricultural fields all the soil samples showed presence of fungal colonies. The fungal colonies were observed on third day whereas full grown fungal colonies were observed on fifth to seventh day at $10^{4}$ serial dilution. Number of colonies in culture plates was counted on third day. The colonies were found diffused with each other on seventh day. From a single soil sample, different fungal colonies were isolated. Out of the sixty soil samples examined for fungal isolation, a total of 2286 fungal colonies were recovered. On an average, 38.1 colonies were isolated from each soil sample. All the sixty soil samples were recorded as $100 \%$ positive for presence of fungal isolates. Out of the 38.1 colonies isolated from soil samples some of the colonies were found morphologically similar to each other on the basis of colony morphology. Some of the fungal colonies were diffused with each other hence could not identified. A total of seven fungal isolates were recovered from organic environment of Durg Chhattisgarh belonging to Genus; Aspergillus, Penicilium, Cladosporium, Fusarium, Rhizopus, Metarhizium and Trichoderma. The Aspergillus was found most predominant soil dwelling fungal species followed by Penicilium, Cladosporium, Fusarium, Rhizopus, Metarhizium and Trichoderma. The percent recovery of fungal isolates of genus Aspergillus was $37.81 \%$ followed by Penicilium, 16.91\%; Cladosporium, 10.94\%; Fusarium, 6.96\%; Rhizopus, 5.22\%; Metarhizium, 5.22\%; Trichoderma, $4.22 \%$ and unidentified was $12.68 \%$. Out of the seven isolates explored from soil samples, four isolates were found to infect ticks and were observed as EPF namely, Fusarium sp,
Rhizopus sp, Metarhizium sp. and Trichoderma $s p$. These fungal isolates were morphologically identified based on colony morphology, shape and colour of colony, mycelium and conidial structure.

The rare occurrence of Metarhizium sp. isolated from natural habitats was observed by Chandler et al., (1997), Bidochka et al., (1998), Meyling and Eilenberg (2005) and Thakur and Sandhu (2010), the findings are in accordance with the present work. In the present study the EPF species isolated from soil belonging to genera Fusarium, Rhizopus, Metarhizium and Trichoderma were recorded.

The present findings were in accordance with Gouli et al., (2013) who also isolated EPF such as Aspergillus, Fusarium and Penicillum. In present investigation, $5.22 \%$ of EPF Metarhizium sp. was observed. Khudhair et al., (2014) reported $18.1 \%$ of EPF belonging to species Metarhizium anisopliae in Iraqi province agro-ecosystems using Galleria mellonella bait trap technique. They reported highest entomopathogenic frequency rate with $55.3 \%$ followed by lowest rate with $17 \%$.

Tkaczuket al., (2015) reported Metarhzium anisopliae and $B$. bassiana formed more colony forming units in soils from organic fields. $M$. anisopliae was the most frequently isolated fungus detected in $92 \%$ of the soil either from organic or conventional fields. Rasheed et al., (2004), Noor Zaman et al., (2012) and Ratna Kumar et al., (2015) isolated fungal genera like Aspergillus, Alternaria, Curvularia, Fusarium, Penicillium and Rhizopus from different crop fields. These observations were similar with present findings. In our investigation among the fungal isolates obtained from the soil samples, the genera Aspergillus and Penicillium were most dominant fungal species. The most common isolates in present 
investigation were Aspergillus fumigatus, A. niger, A. ustus, A. versicolor. Penicillium chrysogenum, $P$. notatum, Cladosporium spherospermum,

Rhizopus

oryzae, Metarhizium majus, Fusarium oxysporum and Trichoderma harzianum.

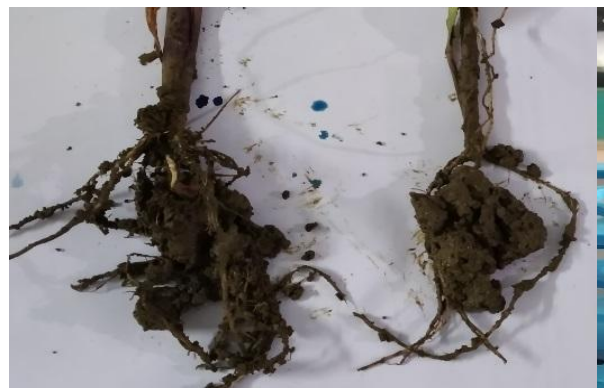

Figure.1 Soil sample collection

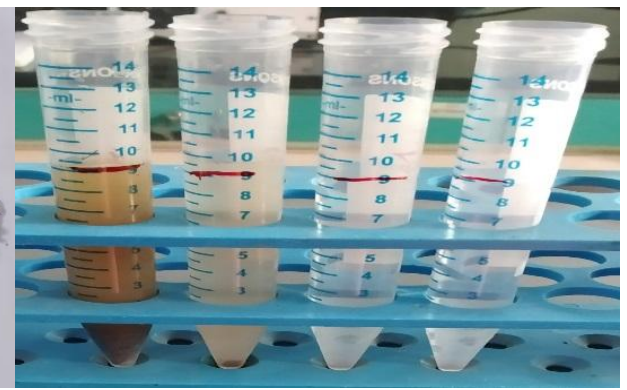

Figure.2 Serial dilution method

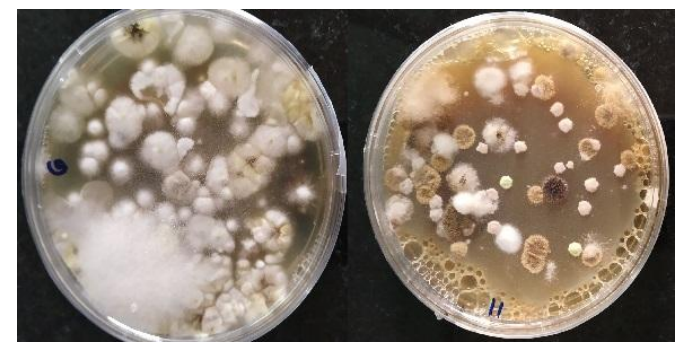

Figure.3 Fungal colonies isolated from soil samples

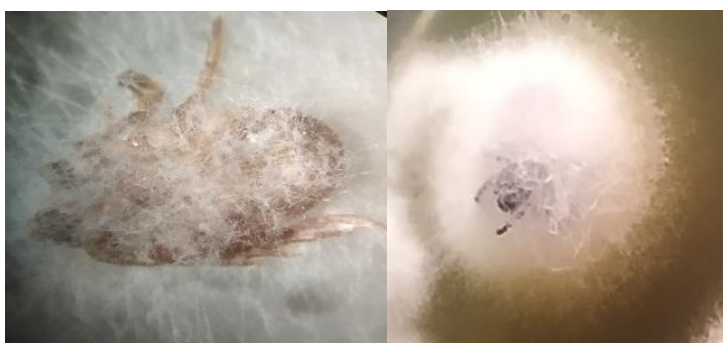

Figure.4 Tick infected with Fusarium and Rhizopus

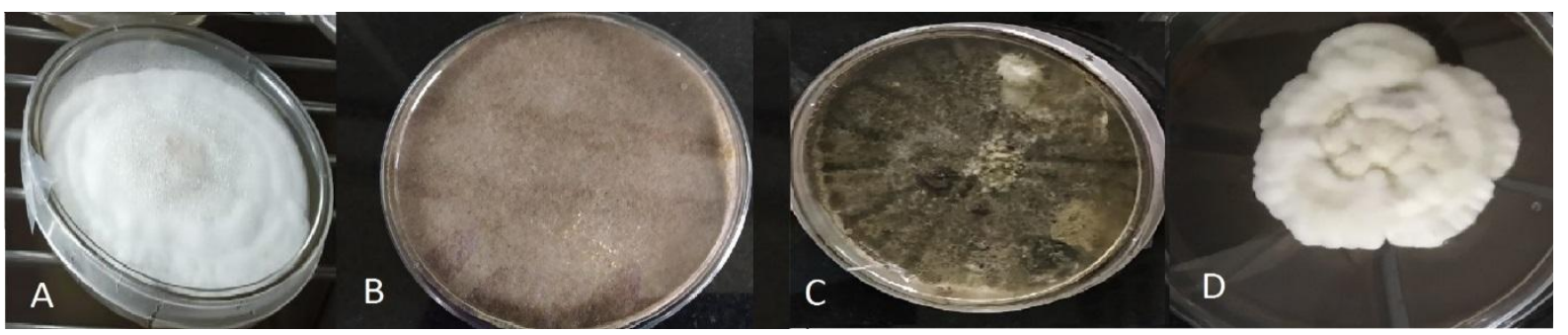

Figure.5 A. Fusarium sp. B. Rhizopus sp. C. Trichoderma sp. D. Metarhizium sp.

Chandini and Rajeshwari (2017), reported that the Penicillium and Aspergillus were the dominant fungi followed by Chaetomium, Trichoderma and Fusarium in Mattavara forest. Fungal frequency of Fusarium oxysporum was noted by them was $33.83 \%$ which was $6.96 \%$ in the present study. Raja et al., (2017) with the similar findings investigated the fungal namely Aspergillus niger, A. clavatus, A. sydowii, As. variabilis, A. fumigatus, Penicillium chrysogenum, Colletotrichum gloeosporioidessenulato, Mucor sp. Rhizopus stolonifer, Rhizopus oryzae, Cunninghamella bertholletiae, Scopulariopsis brumptii, Cladophialophora $s p$. Most of the investigated species are similar with species investigated in the present work. In the present study the rare occurrence of EPF in various cultivated organic agricultural field areas was observed. This may be due to regular use of chemical pesticides, tropical temperature zone and irrigation practices which may be responsible for rare abundance of EPF. The soil moisture has a direct effect on the population of fungi hence, at higher moisture the tolerance and 
colonization is badly affected (Adams et al., 1999). Hummel et al., (2002), in a long-term field study found that the application of certain pesticides significantly reduces the occurrence of EPF in the soil.

\section{References}

Adams, C.P., Bamford, K. M. and Early, M. P. 1990. Principles of Horticulture (3rd Ed.), Utterworth Heineman, pp: 25.

Amy, R. Tuininga, Jessica, L. Miller, Shannon, U. Morath, Thomas, J. Daniels, Richard, C. Falco, Michael Marchese, SadiaSahabi, Dieshia Rosa, and Kirby C. Stafford. 2009. Isolation of Entomopathogenic Fungi from Soils and Ixodesscapularis (Acari: Ixodidae) Ticks: Prevalence and Methods. J Med Entomol. ; 46(3): 557-565.

Bidochka, M.J., Kasperski, J.E. and Wild, G.A.M. 1998. Occurrence of the entomopathogenic fungi Metarhizium anisopliae and Beauveria bassiana in soils from temperate and near northern habitats. Can. J. Bot. 76: 1198-1204.

Chandler, D., Hay, D. and Reid, A. P. 1997. Sampling and occurrence of entomopathogenic fungi and nematodes in UK soils. Applied Soil Ecology 5: 133-141.

Chandini, K.C. and Rajeshwari, N. 2017.Isolation and identification of soil fungi in Mattavara forest, Chikamagalur, Karnataka. Journal of Pharmacognosy and Phytochemistry. 6(5): 721-726.

Eskezia, B. and Desta, A. 2016. Review on the impact of ticks on livestock health and productivity. Journal of Biology, Agriculture and Healthcare. 6(22):1-7.

Gouli, V., Gouli, S., Marcelino, J.A.P., Skinner, M. and Parker, B.L. 2013. Entomopathogenic fungi associated with exotic invasive insect pests in northeastern forests of the USA. Insect,
4: 631-645.

Hummel, R.L., Walegenbach, J. F., Barbercheck, M.E., Kennedy, G.G., Hoyt, G.D., and Arellano C. 2002. Effects of production practices on soilborne entomopathogens in western North Karolina vegetable systems. Environ. Entomol. 31: 84-91.

Khudhair Mohammed W., AlRubeaiHussain F., Khalaf Mohammed Z., Shbar Ali K., HamadBassim S., KhalafHiatham S. 2014. Occurrence and Distribution of Entomopathogenic Fungi in Iraqi AgroEcosystems. Int. J. Entomol. Res. 02 (02) 2014. 117-124.

Lacey, L. A., Frutos, R., Kaya, H. K. and Vail P. 2001. Insect pathogens as biological control agents: Do they have a future? Biological Control; 21: 230-248.

Martins, J. R., Correa, B. L., Cereser, V. H. and Arteche, C. P. 1995. A situation report on resistance to acaricides by the cattle tick Boophilus microplus in the state of Rio Grande do Sul, southern Brazil. In C. Rodriguez, D. Sergio \& H. Fragoso, eds. 3rd International seminary on Animal Parasitology. Acapulco, Guerrero, Mexico. pp. 11-13.

Meyling, N.V. and Eilenberg, J. 2005. Occurrence and distribution of soil borne entomopathogenic fungi within a single organic agroecosystem. Agriculture, Ecosystems and Environment 113: 336-341.

Noor Zaman, Shakil Ahmed. 2012. Survey of Root Rot of Groundnut in Rainfed areas of Punjab, Pakisthan. Afr. J. Biotechnol. 11 (21): 4791-4794.

Raja, M., Praveena, G. and John William, S. 2017. Isolation and Identification of Fungi from Soil in Loyola College Campus, Chennai, India. Int. J. Curr. Microbiol. App. Sci (2017) 6(2): 17891795.

Rasheed, S., Dawar, S. and Ghaffar, A. 2004. Location of fungi in groundnut seed. 
Pak. J. Bot. 36 (3): 663- 668.

Rao, U. B. and Narladkar, B. W. 2017. Role of entomopathogenic fungi in tick control: A Review. Journal of Entomology and Zoology Studies; 6(1): 1265-1269.

Ratna Kumar, P.K., Hemanth, G.P. Shiny Niharika and Samuel, K. Kolli. (2015). Isolation and identification of soil mycoflora in agricultural fields at Tekkali MandalSrikakulam District. Int. J. Adv. Pharmacol., 14 (2): 484-490.

Samish, M., Ginsberg, H. and Glazer, I. 2004. Biological control of ticks. Parasitology, 129: 389-403.

Thakur Rupesh and Sandhu Sardul 2010. Distribution, occurrence and natural invertebrate hosts of indigenous entomopathogenic fungi of Central India. Indian J Microbiol; 50(1):89-96.
Tkaczuk Cezary, KrólAnna, Safaryan Anna Majchrowska and Nicewicz Lukasz. 2014. The occurrence of entomopathogenic fungi in soils from fields cultivated in a conventional and organic system. Journal of Ecological Engineering 15 (4):137-144.

Tuininga Amy R., Miller Jessica L, Morath Shannon U., Daniels Thomas J., Falco Richard C., Marchese Michael, Sahabi Sadia, Rosa Dieshia, and Stafford Kirby C. 2009. Isolation of Entomopathogenic Fungi from Soils and Ixodes scapularis (Acari: Ixodidae) Ticks: Prevalence and Methods. J Med Entomol.; 46(3): 557565.

Waksman, S.A. 1922. A method for counting the number of fungi in the soil. J. Bact.; 7(3): 339-341.

\section{How to cite this article:}

Jumade Pratibha, S. Pal and Sanyal, P.K. 2020. Occurrence and Distribution of Entomopathogenic Fungi in Agricultural Soil of Durg District of Chhattisgarh, India. Int.J.Curr.Microbiol.App.Sci. 9(07): 1150-1155. doi: https://doi.org/10.20546/ijcmas.2020.907.134 